\author{
Public Abstract \\ First Name: Lin \\ Middle Name: \\ Last Name: Zhang \\ Adviser's First Name: Lela \\ Adviser's Last Name: Riley \\ Co-Adviser's First Name: \\ Co-Adviser's Last Name: \\ Graduation Term: SP 2008 \\ Department: Microbiology- Medicine \\ Degree: MS
}

\title{
Title: MICROBIAL PATHOGEN CONTAMINATION IN MOUSE GAMETES AND EMBRYOS
}

To investigate which of the commonly found murine pathogens contaminate mouse gametes and embryos, seven commonly found pathogens in research mice were evaluated in mouse semen, oocyte-cumulus complexes and embryos collected from naturally infected mice in the first part of my study. These seven pathogens are: mouse parvovirus, Helicobacter, Mycoplasma pulmonis, Pasteurella pneumotropica, murine norovirus, mouse hepatitis virus and Theiler's murine encephalomyelitis virus. Five pathogens, mouse parvovirus, Helicobacter, mouse hepatitis virus, murine norovirus and Theiler's encephalomyelitis virus, were found in mouse gametes and embryos. These findings indicate that there is potential risk of transmitting microbial pathogens via cryopreserved gametes and embryos.

The second part of this study focused on evaluating mouse parvovirus and determining the effect of infection in male mice on in vitro fertilization including how it will change fertilization rate, cleavage rate and embryonic development and whether mouse parvovirus transmits to embryos at blastocyst stage. In this study, in vitro fertilization was performed with sperm collected from ICR mice that were inoculated with mouse parvovirus- 1 and oocytes collected from ICR uninfected mice. In control group, IVF was performed by using sperm and oocyte collected from ICR uninfected mice. We did not find mouse parvovirus-1 infection in male mice transmitted to in vitro produced embryos and there was no effect on fertilization rate, cleavage rate and embryonic development during in vitro fertilization. 